\title{
Publisher's Note: Uncertainty decomposition method and its application to the liquid drop model
} [Phys. Rev. C 93, 034310 (2016)]

Cenxi Yuan

(Received 11 March 2016; published 25 March 2016)

DOI: 10.1103/PhysRevC.93.039901

This paper was published online on 8 March 2016 with an error in the text of the abstract. The twelfth sentence in the abstract should read as "The light and heavy nuclei are indeed distributed mostly inside the distributions of the systematic and statistical uncertainties, respectively." The paper has been corrected as of 10 March 2016. The text is correct in the printed version of the journal. 